\title{
Early Data on Long-term Impact of Inotersen on Quality-of-Life in Patients with Hereditary Transthyretin Amyloidosis Polyneuropathy: Open-Label Extension of NEURO-TTR
}

\author{
Aaron Yarlas (D) - Andrew Lovley · Kristen McCausland • \\ Duncan Brown · Montserrat Vera-Llonch • Isabel Conceição • \\ Chafic Karam · Sami Khella · Laura Obici · Márcia Waddington-Cruz
}

Received: May 3, 2021 / Accepted: July 15, 2021 / Published online: August 5, 2021

(C) The Author(s) 2021

\section{ABSTRACT}

Introduction: Patients with hereditary transthyretin amyloidosis associated with polyneuropathy (ATTRv-PN) experience deterioration in health-related quality of life (HRQOL) as the disease progresses. Findings from the randomized placebo-controlled phase III NEURO-TTR study showed treatment benefit of inotersen, an antisense oligonucleotide, for preserving or improving HRQOL after 65 weeks of treatment. The current analysis examines longitudinal

\author{
A. Yarlas $(\bowtie) \cdot$ A. Lovley $\cdot$ K. McCausland \\ QualityMetric, 1301 Atwood Avenue, Suite 316E, \\ Johnston, RI 02919, USA \\ e-mail: ayarlas@qualitymetric.com \\ D. Brown · M. Vera-Llonch \\ Ionis Pharmaceuticals, Boston, MA, USA \\ I. Conceição \\ Centro Hospitalar Universitário Lisboa Norte, \\ Hospital de Santa Maria and Faculdade de Medicina, \\ Lisbon, Portugal \\ C. Karam · S. Khella \\ University of Pennsylvania, Philadelphia, PA, USA \\ L. Obici \\ Amyloidosis Research and Treatment Center, \\ Fondazione IRCCS Policlinico San Matteo, Pavia, \\ Italy \\ M. Waddington-Cruz \\ Federal University of Rio de Janeiro, National \\ Amyloidosis Referral Center, CEPARM, Rio de \\ Janeiro, Brazil
}

trends in specific aspects of HRQOL, including polyneuropathy symptoms, daily activities, and physical, role, and social functioning in patients with ATTRv-PN receiving long-term treatment in a follow-up open-label extension (OLE) study.

Methods: One-hundred thirty-five patients with ATTRv-PN were enrolled in an ongoing 5 -year OLE study following completion of NEURO-TTR. Eighty-five patients received continuous weekly treatment with inotersen in both studies (inotersen-inotersen group), while 50 patients switched from placebo to inotersen at OLE study baseline (placebo-inotersen group). Descriptive analyses of changes in domain scores and item responses through week 104 of the OLE study were conducted for measures of neuropathy-related and generic HRQOL: Norfolk QOL-Diabetic Neuropathy (DN) questionnaire and SF-36v2 ${ }^{\circledR}$ Health Survey (SF-36v2), respectively.

Results: For both inotersen-inotersen and placebo-inotersen groups, all Norfolk QOL-DN and most SF-36v2 domain scores remained stable from OLE baseline through week 104. Differences in HRQOL between the two groups at OLE baseline were sustained through week 104. Analysis of item responses from NEUROTTR baseline to OLE study week 104 (170 weeks) for the inotersen-inotersen group found no notable increases in the proportion of patients reporting substantial impairments across a wide 
variety of symptoms, daily activities, and functioning.

Conclusion: Long-term treatment with inotersen preserved HRQOL for patients with ATTRv-PN for periods of up to 3 years. The gap in HRQOL between those who had previously received inotersen or placebo in NEURO-TTR did not close by week 104 of the OLE phase, indicating the importance of early treatment for maintaining HRQOL in these patients.

Trial Registration: ClinicalTrials.gov Identifiers NCT01737398 for NEURO-TTR study; NCT02175004 for OLE study

Infographic: 


\section{Early Data on Long-term Impact of Inotersen on Quality-of-Life in Patients with Hereditary Transthyretin Amyloidosis Polyneuropathy: Open-label Extension of NEURO-HTR \\ Aaron Yarlas, Andrew Lovley, Kristen McCausland, Duncan Brown, Montserrat Vera-Llonch, Isabel Conceição, Chafic Karam, Sami Khella, Laura Obici, Márcia Waddington-Cruz}

NEURO-TTR
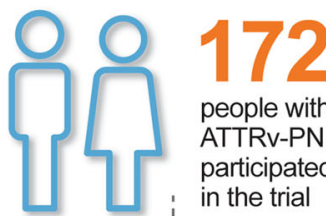

people with ATTRv-PN participated in the trial

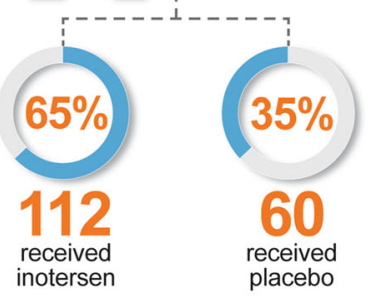

OLE Trial (up to 5 years)
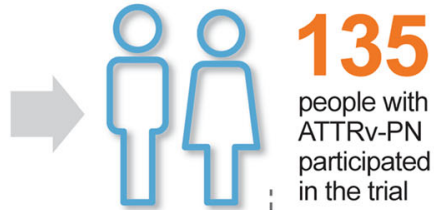

people with ATTRv-PN participated in the trial

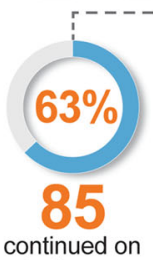

continued on inotersen
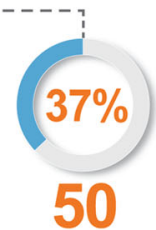

were switched

were switched

to inotersen
(from placebo)

Interim analysis included data at up to 2 years

\section{6 week NEURO-TTR}

Patients with this disease who were treated with the drug inotersen for 66 weeks maintained their quality of life, while patients who received placebo showed continued worsening.

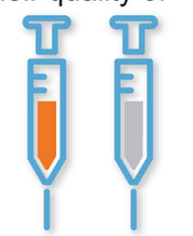

\section{extension study}

For all patients, regardless of previous treatment (inotersen or placebo), most aspects of quality of life did not change throughout the two year extension study, showing that inotersen can preserve quality of life of these patients for up to two-to-three years.

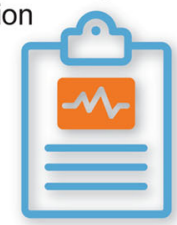

\section{Conclusion:}

Long-term treatment with inotersen preserved health-related quality of life (HRQOL) for patients with ATTRv-PN for periods of up to three years. The gap in $\mathrm{HRQOL}$ between those who had previously received inotersen or placebo in NEURO-TTR did not close by week

104 of the OLE phase, indicating the importance of early treatment for maintaining HRQOL in these patients.

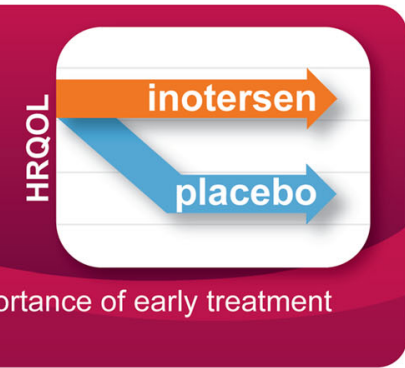

This infographic represents the opinions of the authors. For a full list of declarations, including funding and author disclosure statements, please see the full text online. (C) The authors, CC-BY-NC 2021.

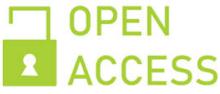




\section{PLAIN LANGUAGE SUMMARY}

Hereditary transthyretin amyloidosis with polyneuropathy is a rare disease that causes damage to nerves in the limbs, leading to pain, numbness, loss of sensitivity, and muscle weakness, with eventual loss of the ability to walk (i.e., patients require a wheelchair or are bedridden). As the disease progresses, patients' quality of life, including their ability to engage in everyday activities, socialize with others, work, and live independently, continually worsens. In a recent clinical trial (the NEUROTTR study), patients with this disease randomized to receive the drug inotersen for 66 weeks maintained their quality of life, while patients randomized to receive a placebo showed continued worsening. All patients completing the NEURO-TTR study could participate in an extension study during which all patients knowingly received inotersen for up to 5 years. We examined quality of life in patients through the first 2 years of this extension study. For all patients, regardless of previous treatment (inotersen or placebo), most aspects of quality of life did not change throughout the 2-year extension study, showing that inotersen can preserve quality of life of these patients for up to 2-3 years. However, while quality of life in patients who had received placebo in the NEURO-TTR study did not get worse during the extension study, it also did not improve to match that of patients who received inotersen during the NEURO-TTR study. This finding shows the importance of treating these patients with inotersen as early as possible to preserve their quality of life before it substantially deteriorates.

Keywords: Clinical trial; Hereditary transthyretin amyloidosis; Inotersen; Openlabel extension trial; Physical function; Polyneuropathy; Quality of life; Rare disease

\section{Key Summary Points}

\section{Why carry out this study?}

Results from the phase 3 NEURO-TTR study found that 65 weeks of treatment with inotersen (Tegsedi ${ }^{\mathrm{TM}}$ ) led to improvements, relative to placebo, in the health-related quality of life (HRQOL) of patients with hereditary transthyretin amyloidosis associated with polyneuropathy (ATTRv-PN).

Because the long-term impact of inotersen on HRQOL for these patients has not yet been examined, the purpose of the current study was to evaluate longitudinal trends in HRQOL for patients in the NEURO-TTR trial who subsequently enrolled in an open-label extension (OLE) study for up to 2 years.

\section{What was learned from the study?}

The results of this interim analysis of data from the OLE study provide preliminary evidence that inotersen treatment stabilizes many aspects of HRQOL, including activities of daily living and physical, role, and social functioning, for up to 2-3 years, although due to limitations from the small sample size, further research within a larger patient sample is warranted.

Because previous studies observed no additional safety concerns or increased toxicity in patients exposed to inotersen for up to 5 years, inotersen may be a promising long-term treatment option for these patients.

While deterioration of most aspects of patients' HRQOL was arrested by inotersen, it was not reversed, which highlights the importance of early diagnosis and treatment of this disease. 


\section{DIGITAL FEATURES}

This article is published with digital features, including an infographic and plain language summary, to facilitate understanding of the article. To view digital features for this article go to https://doi.org/10.6084/m9.figshare. 14987385.

\section{INTRODUCTION}

Hereditary transthyretin (ATTRv) amyloidosis is a rare systemic, progressive, life-threatening disease caused by mutations in the genetic encoding of transthyretin (TTR) protein, which is primarily synthesized in the liver $[1,2]$. More than 140 TTR genetic mutations have been identified worldwide [3]. These mutations result in TTR misfolding and accumulation as amyloid deposits in tissues and organs, resulting in damage to the nervous system and multiple organs [4]. Patients with ATTRv amyloidosis commonly experience injury to the somatic and autonomic nerve fibers.

Patients with ATTRv amyloidosis associated with polyneuropathy (ATTRv-PN) experience a substantial burden on their health-related quality of life (HRQOL) [5-7], which further deteriorates as the disease progresses $[8,9]$.

Results from the phase 3 NEURO-TTR study found that 65 weeks of treatment with inotersen (Tegsedi ${ }^{\mathrm{TM}}$ ), a 2'-O-methyoxyethyl-modified antisense oligonucleotide that reduces TTR production, led to improvements, relative to placebo, in neuropathic impairment of patients with ATTRv-PN [11]. Data from the clinical trial also supported preservation or improvement in HRQOL of patients receiving inotersen for 65 weeks compared to significant deteriorations in HRQOL for patients receiving placebo for this duration. As assessed by changes in scores from baseline to week 66 on the Norfolk QOL-DN, a patient-reported assessment of neuropathyspecific HRQOL, patients receiving inotersen showed relative improvement on total score [10] as well as on domains capturing the impact of neuropathy on activities of daily living (ADL), physical functioning associated with large fiber neuropathy, and neuropathic symptoms [12]. In addition, change in scores over this period on the SF-36v2 ${ }^{\circledR}$ Health Survey (SF-36v2), a patient-reported assessment of generic HRQOL, showed relative improvement for patients receiving inotersen on global physical HRQOL, as captured by the physical component summary (PCS) score [10], as well as on domains evaluating physical functioning, role limitations due to both physical and emotional health problems, pain, and social functioning [12]. Furthermore, descriptive analyses of responses to items on these two HRQOL measures between treatment arms showed consistent benefits of inotersen on specific impacts on neuropathic symptoms, activities of daily living, and physical, role, and social functioning of patients following 65 weeks of treatment [12].

Patients who completed the NEURO-TTR study were eligible to receive inotersen for up to 5 years in a follow-up open-label extension (OLE) study. Analysis of early data from the OLE study showed maintenance of stabilization for patients who received inotersen during the NEURO-TTR study (the inotersen-inotersen group) and induction of stabilization for patients who received placebo in the NEUROTTR study (the placebo-inotersen group), in neuropathic impairment and global summary measures of HRQOL (Norfolk QOL-DN total score and SF-36v2 PCS score) after receiving 3.25 years or 2 years of continuous treatment with inotersen at week 104 of the OLE study, respectively [13]. Patients in the placebo-inotersen group still showed greater neuropathic impairment after 2 years of treatment with inotersen at week 104 of the OLE study than did patients in the inotersen-inotersen group, showing the importance of early treatment intervention. However, initiation of inotersen for patients in the placebo-inotersen group did result in stabilization of these outcomes, indicating that patients who receive intervention later in the disease course still experience a treatment benefit.

While these preliminary findings speak to the long-term effectiveness of inotersen for stabilizing global measures of HRQOL, its impacts on specific aspects of neuropathyspecific and generic HRQOL over this extended 
duration have not previously been examined. The purpose of the current study was to evaluate longitudinal trends in HRQOL by examining changes in mean domain scores for the Norfolk QOL-DN and SF-36v2 by treatment group (inotersen-inotersen vs. placebo-inotersen) through the first 104 weeks ( 2 years) of the OLE study and examining changes in responses to selected items from these measures for patients in the inotersen-inotersen group over the 170-week observation period spanning the full NEURO-TTR study and the first 104 weeks of the OLE study.

\section{METHODS}

\section{Study Design and Treatment}

The NEURO-TTR study (ClinicalTrials.gov Identifier: NCT01737398) was a phase 3, multinational, randomized, double-blind, clinical trial for adult patients with ATTRv-PN. Patients were eligible to enroll in the NEUROTTR study if they were diagnosed with ATTRvPN, aged 18-82 years, classified as having either Stage 1 (ambulatory without assistance) or Stage 2 (ambulatory with assistance) neurologic disease severity (based on Coutinho et al.'s [1980] staging [14]), had a Neuropathy Impairment Score (NIS) of 10 to 130, a TTR mutation determined by genotyping, documented amyloid deposits determined by biopsy, no clinically significant abnormalities in screening laboratory values, a Karnofsky performance status score $>50$, no other causes of polyneuropathy, no previous liver transplantation, and no heart failure of NYHA class III or higher [10]. Enrolled patients were randomized in a 2:1 ratio to receive $300 \mathrm{mg}$ subcutaneous inotersen or matching placebo once weekly for 65 weeks. A more detailed description of the NEURO-TTR study sample and design has been previously published [10]. The Norfolk QOL-DN and SF36v2 were administered to patients at baseline, week 35, and week 66 visits of the NEURO-TTR study, which will be referred to as the NEUROTTR observation period.

Patients who had satisfactory completion of dosing and end-of-treatment efficacy assessments, experienced no significant tolerability issues and had satisfactory compliance with the NEURO-TTR study protocol were eligible to enroll in the follow-up OLE study (ClinicalTrials.gov Identifier: NCT02175004), during which each patient received $300 \mathrm{mg}$ subcutaneous inotersen once weekly for up to 260 weeks (5 years). The Norfolk QOL-DN and SF-36v2 were administered to patients at OLE baseline and at follow-up visits at each subsequent 26-week period through week 260 . The current analysis, which is based on an interim data set from 31 May 2018, includes data from visits at OLE baseline, week 26, week 52, week 78 , and week 104 visits, which will be referred to as the OLE observation period. A more detailed description of the OLE study sample and design has been published elsewhere [13].

The 170-week period spanning the NEUROTTR observation period and the OLE observation period will be referred to as the cumulative observation period.

\section{Ethical Standards}

The protocols of both NEURO-TTR and OLE studies were approved by the relevant institutional review boards or local ethics committees and regulatory authorities. Both studies were conducted in accordance with Good Clinical Practice guidelines of the International Conference on Harmonization and the principles of the Declaration of Helsinki. All patients provided written informed consent to participate in the trial.

\section{Study Assessments}

The 35-item Norfolk QOL-DN is a self-reported measure of neuropathy-related HRQOL [15]. While it was originally developed for patients with $\mathrm{DN}$, its reliability and validity have been established for assessment of neuropathy-related HRQOL in patients with ATTRv-PN [16].

The Norfolk QOL-DN total score is calculated as the sum of responses across all 35 items, with scores ranging from -4 to 136 . Additionally, scores can be calculated for each of five domains capturing outcomes associated with nerve fiber 
damage: ADL (5 items, with a score range of 0 to 20); autonomic neuropathy (3 items, 0 to 12 ), large fiber neuropathy/physical functioning (15 items, -4 to 56); small fiber neuropathy (4 items, 0 to 16 ); neuropathic symptoms (8 items, 0 to 32 ). For total and all domain scores, higher values indicate worse HRQOL.

The 36-item SF-36v2 is a self-reported measure of generic HRQOL [17]. The SF-36v2 has been previously used to assess treatment benefit on HRQOL in patients with ATTRv-PN [18]. The survey is available in acute form (1-week recall period) and standard form (4-week recall period); the standard form was administered at all assessments. Responses to SF-36v2 items can be used to compute scores for eight domains of HRQOL: physical functioning, role limitations due to physical health (role-physical), bodily pain, perception of general health (general health), vitality, social functioning, role limitations due to emotional health (role-emotional), and mental health. Two summary scores-PCS, which captures global physical HRQOL, and the Mental Component Summary (MCS), which captures global mental HRQOL-are calculated as weighted sums of scores from all eight domains. All SF-36v2 domains and summary scores are expressed as $T$ scores using normbased methods, standardized to a mean of 50 and a standard deviation of 10 in the general population. For both summary and all domain scores, higher values indicate better HRQOL.

\section{Statistical Analysis}

All analyses described here were post hoc and descriptive. No hypotheses were tested; no inferential statistics or $p$-values were calculated. Analyses were based on the sample of patients who enrolled in the OLE study and were conducted separately for inotersen-inotersen and placebo-inotersen groups. Descriptive statistics were based on observed responses only; no imputation method was used for missing values.

For both inotersen-inotersen and placeboinotersen groups, mean changes in observed scores for all Norfolk QOL-DN and SF-36v2 domains from NEURO-TTR study baseline were calculated at all subsequent visits of the cumulative observation period, while mean changes in observed scores from OLE study baseline were calculated for all subsequent visits of the OLE observation period. Mean changes in domain scores from OLE baseline to week 104 were examined for all observed patients as well as for the subgroup of patients who completed the week 104 visit. The magnitude of mean changes between patient groups and the longitudinal patterns of mean changes across visits within each group were evaluated for each domain using descriptive analyses and visual examination of graphical plots for means and standard errors.

To provide a real-world context for assessing the impact of inotersen on everyday functioning across a period of over 3 years, responses to selected items from the Norfolk QOL-DN and SF-36v2 were analyzed for the inotersen-inotersen group (it was determined a priori that the sample sizes of the placebo-inotersen group, particularly at later visits of the OLE study, were not large enough to support interpretable comparisons of changes in within-group proportions across visits). All items selected in the analyses concern specific impacts of the disease and its treatment on neuropathic symptoms, ability to participate in daily activities, and physical, role, and social functioning. Patients' responses were analyzed for 19 items from among those on each of the five domains of the Norfolk QOL-DN and for 16 items from among those on the physical functioning, role-physical, bodily pain, and social functioning domains of the SF-36v2 that were analyzed previously for the NEURO-TTR study [12] (content for each of the selected Norfolk QOL-DN and SF-36v2 items is presented below in Tables 4 and 5, respectively). Response choices for each selected item were dichotomized to indicate whether a patient experienced either 'substantial impairment' or 'not substantial impairment' on the concept captured by the item. All examined items from the Norfolk QOL-DN used the same five response options: 'not a problem,' 'very mild problem,' 'mild problem,' 'moderate problem,' and 'severe problem.' For each item, patients who selected 'moderate problem' or 'severe problem' were 
classified as having substantial impairment, while those who selected 'not a problem,' 'very mild problem,' or 'mild problem' were classified as not having substantial impairment. The items from the SF-36v2 physical functioning domain all use the same three response options: 'not limited at all,' 'limited a little,' and 'limited a lot.' Patients who selected 'limited a lot' were classified as having substantial impairment, while patients who selected 'limited a little' or 'not limited at all' were classified as not having substantial impairment. Items on the SF-36v2 role-physical domain all use the same five response options: 'all of the time,' 'most of the time,' 'some of the time,' 'a little of the time,' and 'none of the time.' Patients who selected 'all of the time' or 'most of the time' were classified as having substantial impairment, while those selecting 'some of the time,' 'a little of the time,' or 'none of the time' were considered to not have substantial impairment. The single items selected from the SF-36v2 bodily pain and social function domains-"How much did pain interfere with your normal work, including both work outside the home and housework?" and "During the past 4 weeks, to what extent has your physical health or emotional problems interfered with your normal social activities with family, friends, neighbors, or groups?", respectively-used the same five response options: 'extremely,' 'quite a bit,' 'moderately,' 'a little bit,' and 'not at all.' Patients who selected 'extremely' or 'quite a bit' were classified as having substantial impairment, while those selecting 'moderately,' 'a little bit,' or 'not at all' were considered to not have substantial impairment.

The percentages of patients whose observed item-level response indicated a substantial level of impairment was calculated for the inoterseninotersen group for the cumulative study period. Proportions of patients indicating substantial impairment were evaluated longitudinally for each item using descriptive analyses and visual examination of graphical plots for percentages and standard errors for the cumulative observation period.

\section{RESULTS}

\section{OLE Study Population}

Of the 139 patients who completed the NEUROTTR study (i.e., $80.8 \%$ of the 172 patients who received at least one dose of trial regimen during the study [10]), 135 (97.1\%) enrolled in the OLE study, with 85 patients (of 87 completers) in the inotersen-inotersen group and 50 patients (of 52 completers) in the placeboinotersen group. As shown in Table 1, demographics and disease characteristics at OLE study baseline were generally well balanced between the inotersen-inotersen and placeboinotersen groups. At the time of the data cut for this interim dataset, 41 patients in the inotersen-inotersen and 19 patients in the placeboinotersen groups had completed the week 104 visit, with 58 and 35 patients ongoing in the study within each group, respectively. Detailed descriptions regarding patients' treatment exposure, dose pause, and use of concomitant medications have been published elsewhere [13].

\section{Mean Change in Domain Scores}

\section{Norfolk QOL-DN domains}

Mean changes in observed Norfolk QOL-DN domain scores from NEURO-TTR baseline to each subsequent assessment in the NEURO-TTR and OLE studies are presented in Fig. 1i-v.

Similar patterns of mean changes can be seen for the three domains-ADL (Fig. 1i), large fiber neuropathy/physical functioning (Fig. 1iii), and neuropathic symptoms (Fig. 1v)-for which statistically significant treatment benefits of inotersen were observed in the NEURO-TTR study [12]. Specifically, for each of these domains, mean changes in the inotersen-inotersen group remained relatively stable, and close to 0 , throughout the cumulative observation period. In contrast, for the placebo-inotersen group, scores deteriorated during the NEURO-TTR observation period but then stabilized in the OLE observation period. The magnitude of differences in domain scores between the patient groups observed at the final (week 
Table 1 Baseline demographics and disease characteristics in the OLE study $(n=135)$

\begin{tabular}{lll}
\hline Patient characteristics & $\begin{array}{c}\text { Inotersen- } \\
\text { inotersen } \\
(\boldsymbol{n}=\mathbf{8 5})\end{array}$ & $\begin{array}{l}\text { Placebo- } \\
\text { inotersen } \\
(\boldsymbol{n}=\mathbf{5 0})\end{array}$ \\
\hline $\begin{array}{l}\text { Age, mean (SD; } \\
\text { min-max), years }\end{array}$ & $\begin{array}{c}60.3(11.9 ; \\
29-79)\end{array}$ & $\begin{array}{c}60.5(14.6 ; \\
29-82)\end{array}$ \\
$\begin{array}{l}\text { Male, } n \text { (\%) } \\
\text { Neuropathy stage [14], } n(\%)\end{array}$ & $35(70.0)$ \\
$\begin{array}{l}\text { Stage 1 } \\
\text { Stage 2 }\end{array}$ & $55(64.7)$ & $28(56.0)$ \\
$\begin{array}{l}\text { Stage 3 } \\
\text { Val30Met TTR } \\
\text { mutation, }{ }^{\mathrm{a}} n \text { (\%) }\end{array}$ & $39(31.8)$ & $21(42.0)$ \\
$\begin{array}{l}\text { Prior use of stabilizers, }{ }^{\mathrm{a}, \mathrm{b}} \\
n \text { (\%) }\end{array}$ & $53(62.4)$ & $27(5.0)$ \\
$\begin{array}{l}\text { Duration of ATTRv-PN } \\
\text { symptoms, mean (SD), } \\
\text { months }\end{array}$ & $79.7(49.0)$ & $82.0(55.8)$ \\
$\begin{array}{l}\text { Patients with } \\
\text { cardiomyopathy, }{ }^{a} n \text { (\%) }\end{array}$ & $59(69.4)$ & $30(60.0)$ \\
\hline
\end{tabular}

$A T T R v-P N$ hereditary transthyretin amyloidosis with polyneuropathy, $O L E$ open-label extension, $P N$ polyneuropathy, $S D$ standard deviation, TTR transthyretin

a Based on assessment at NEURO-TTR study entry

b Prior stabilizer use includes tafamidis and/or diflunisal

66) visit of the NEURO-TTR study was relatively unchanged during the OLE observation period, indicating the patients in the placebo-inotersen group were neither catching up to nor falling further behind the patients in inotersen-inotersen group, but rather they maintained the same trajectory of minimal change. For the autonomic neuropathy domain (Fig. 1ii), the lack of progression and differences between patient groups that was observed during the NEURO-TTR observation period continued throughout the OLE observation period. For the small fiber neuropathy domain (Fig. 1iv), patients in the inotersen-inotersen group continue to show no progression (all mean change $<1$ point), while patients in the placebo-inotersen group show some progression during the OLE observation period, with increases of 1.9 points at week 52 and 1.4 points at week 104. However, at week 104, the difference in mean change between groups remains $<$ 1 point.

Domain scores were relatively stable for each group throughout the OLE observation period, as indicated by the small magnitude of mean changes from OLE study baseline to week 104 that are reported in Table 2. For both the inotersen-inotersen and placebo-inotersen groups, these changes in mean scores were relatively small for all domains (approximately 2 points for ADL and larger fiber neuropathy/physical functioning and $\leq 1.2$ points for the other domains). This pattern persisted for all patients at each of the two visits as well as for the subgroup of patients who completed the week 104 visit. For the week 104 completers in both groups, the $95 \%$ confidence intervals for mean change in scores include the value of 0 for all domains except ADL, which provides evidence supporting the stability of these scores in the OLE observation period.

\section{SF-36v2 domains}

Mean changes in observed Norfolk QOL-DN domain scores from NEURO-TTR baseline to each subsequent assessment in the NEURO-TTR and OLE studies are presented in Fig. 2i-viii.

Similar patterns of mean changes can be seen for four domains-physical functioning (Fig. 2i), role-physical (Fig. 2ii), bodily pain (Fig. 2iii), and role-emotional (Fig. 2vii)-for which statistically significant treatment benefits of inotersen were observed in the NEURO-TTR observation period [12]. Specifically, for each of these domains, mean changes in the inoterseninotersen group remain relatively stable, and close to 0 , throughout the cumulative observation period (with the exception of bodily pain, which shows an approximately 3-point improvement at the week 35 visit of the NEURO-TTR study that is maintained throughout the cumulative observation period). For the placebo-inotersen group, scores deteriorated during the NEURO-TTR observation period, but then stabilized during the OLE observation period (also with the exception of bodily pain, 

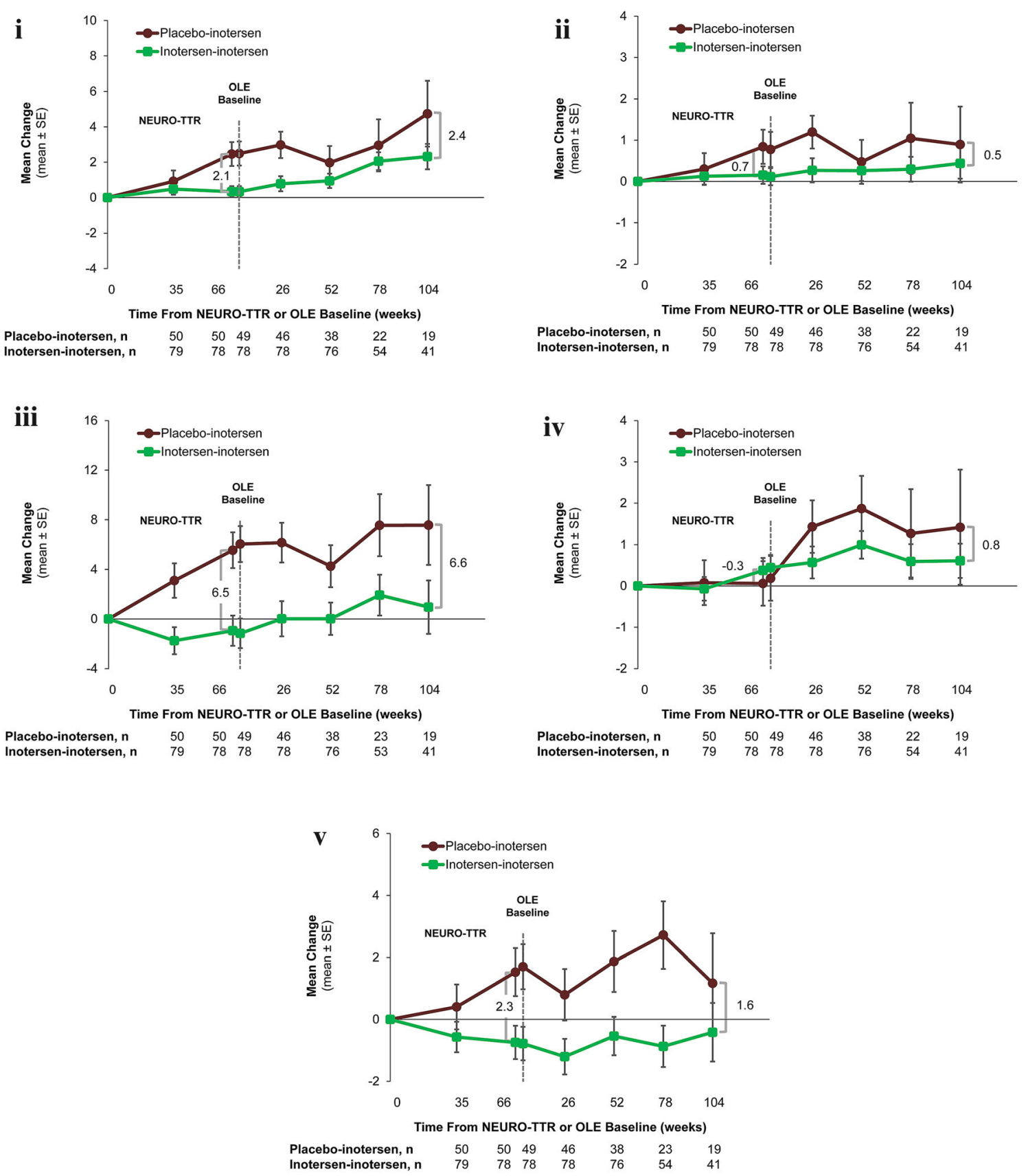

Fig. 1 Mean change in Norfolk QOL-DN domain scores from NEURO-TTR baseline through week 104 of the open-label extension study. i. Activities of daily living domain. ii. Autonomic neuropathy domain. iii. Large fiber

which showed an approximately 3-point improvement at the week 26 visit of the OLE study that is maintained through week 104). The similar magnitudes of differences for neuropathy/physical functioning domain. iv. Small fiber neuropathy domain. v. Neuropathic symptoms domain. $O L E$ open-label extension, $S E$ standard error

domain scores between the patient groups observed at the end of the NEURO-TTR and OLE observation periods indicate a parallel trajectory for the two groups. 
Table 2 Mean change in Norfolk QOL-DN domain scores from OLE baseline to week 104

\begin{tabular}{llcllc}
\hline Domains & \multicolumn{2}{l}{ All observed scores, mean } & & \multicolumn{2}{l}{ Week 104 completers, mean (95\% CI) } \\
\cline { 2 - 3 } \cline { 6 - 6 } & $\mathbf{I}-\mathbf{I}(\boldsymbol{n}=\mathbf{7 8})$ & P-I $(\boldsymbol{n}=\mathbf{4 9 )}$ & & $\mathbf{I}-\mathbf{I}(\boldsymbol{n}=\mathbf{4 1})$ & P-I $(\boldsymbol{n}=\mathbf{1 9})$ \\
\hline ADL & 2.0 & 2.3 & & $1.9(0.6,3.1)$ & $2.1(0.2,4.0)$ \\
Autonomic neuropathy & 0.3 & 0.1 & & $0.4(-0.2,1.0)$ & $-0.2(-1.1,0.8)$ \\
Large fiber neuropathy/PF & 2.1 & 1.5 & & $2.5(-1.2,6.2)$ & $0.5(-3.5,4.4)$ \\
Small fiber neuropathy & 0.2 & 1.2 & & $0.2(-0.6,1.0)$ & $1.3(-0.9,3.4)$ \\
Neuropathic symptoms & 0.4 & -0.5 & & $0.2(-1.6,2.1)$ & $-1.4(-3.4,0.6)$ \\
\hline
\end{tabular}

$A D L$ activities of daily living, $C I$ confidence interval, $I-I$ inotersen-inotersen group, $O L E$ open-label extension, $P-I$ placeboinotersen group, $P F$ physical functioning

Trajectories for mean changes in other SF$36 \mathrm{v} 2$ domains across the cumulative observation period differed from those described above. A statistically significant treatment benefit of inotersen was observed during the NEURO-TTR observation period for the social functioning domain [12], but while stabilization was maintained throughout the OLE observation period for patients in the inotersen-inotersen group, the placebo-inotersen group continued to show further deterioration during the OLE observation period, with the difference in mean change scores between groups doubling from 4.0 to 8.3 points following a substantial drop from week 52 to week 78 (Fig. 2vi). For general health (Fig. 2iv) and vitality domains (Fig. 2v), for which there were no statistically significant treatment differences at week 66 of the NEUROTTR observation period [12], a similar pattern was found such that the inotersen-inotersen group remained stable during the OLE observation period, while the placebo-inotersen group showed declines during this period, with the largest drop-off again observed between week 52 to week 78 visits, with the differences between groups more than tripling from week 66 of the NEURO-TTR observation period to week 104 of the OLE observation period (1.6 to 6.2 points for general health; 1.7 to 5.6 points for vitality). Finally, the mental health domain scores in both groups were stable throughout the cumulative observation period (Fig. 2viii).

The relative stability of scores for all domains for the inotersen-inotersen group through the OLE observation period and the differences in trajectories among domains for the placeboinotersen group during this period are shown in Table 3. For the inotersen-inotersen group, the change in mean scores during the OLE observation period were relatively small (no decrease $>2$ points for any domain) among all observed patients, while for the subgroup of week 104 completers, the 95\% confidence intervals for mean change include the value of 0 for all domains. These same findings were observed for the placebo-inotersen group on the majority of domains, although large decreases in scores were observed during the OLE observation period for general health, vitality, and social functioning domains when considering all observed patients.

\section{Proportion of Patients Showing Substantial Impairment Based on Item Responses}

\section{Norfolk QOL-DN Items}

The percentages of patients in the inoterseninotersen group indicating substantial impairment for each of the 19 selected Norfolk QOLDN items, based on all observed item responses at each assessment in the cumulative observation period, are presented in Fig. 3i (five items from the ADL domain), Fig. 3ii (six items from the large fiber neuropathy/physical functioning domain), Fig. 3iii (four items from the small fiber neuropathy domain), and Fig. 3iv (three items from the autonomic neuropathy domain and one item from the neuropathic symptoms 

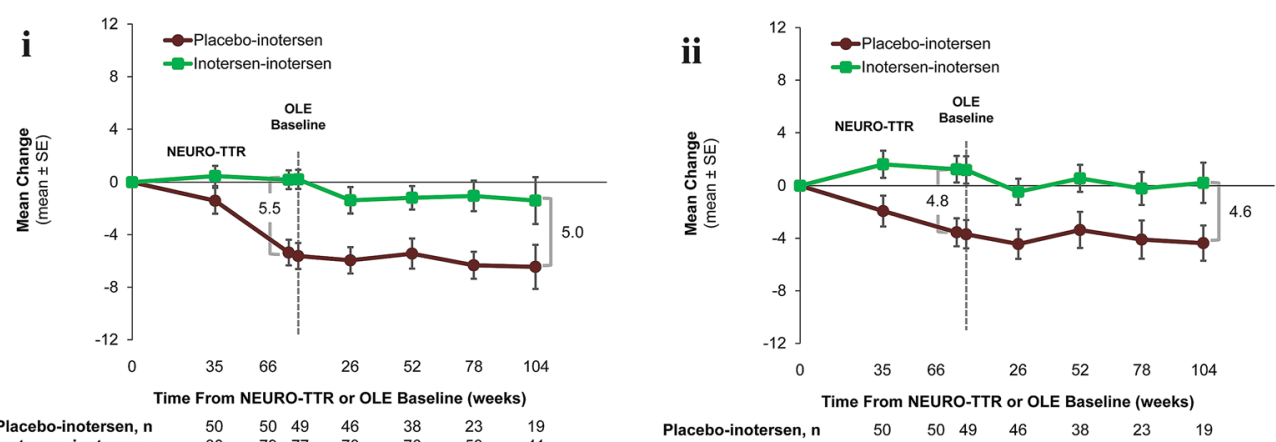

$\begin{array}{llllllll}\text { Placebo-inotersen, } \boldsymbol{n} & 50 & 50 & 49 & 46 & 38 & 23 & 19 \\ \text { Inotersen-inotersen, } \mathbf{n} & 80 & 79 & 77 & 78 & 76 & 53 & 41\end{array}$
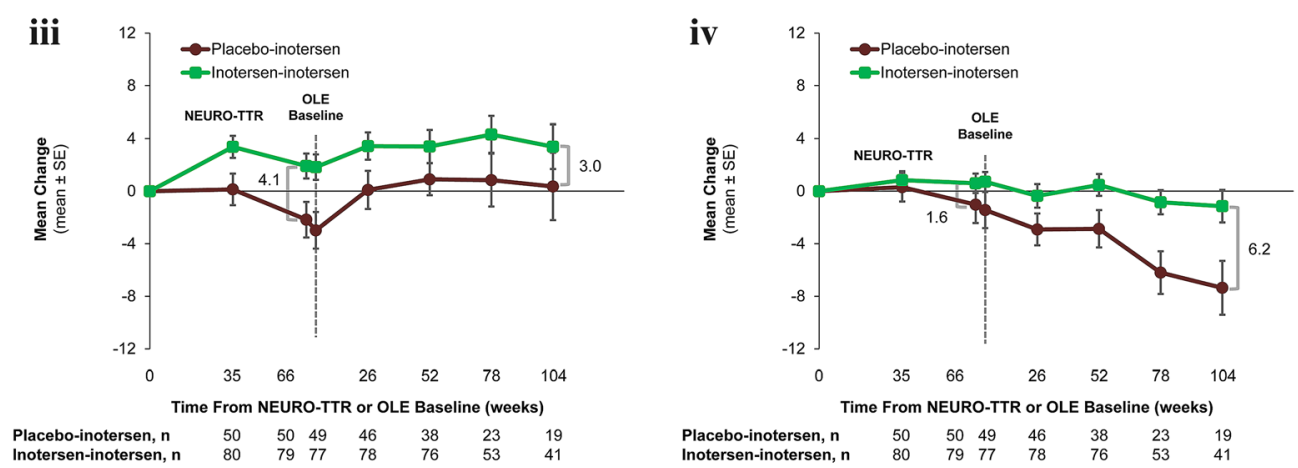

$\begin{array}{llllllll}\text { Placebo-inotersen, } \boldsymbol{n} & 50 & 50 & 49 & 46 & 38 & 23 & 19\end{array}$
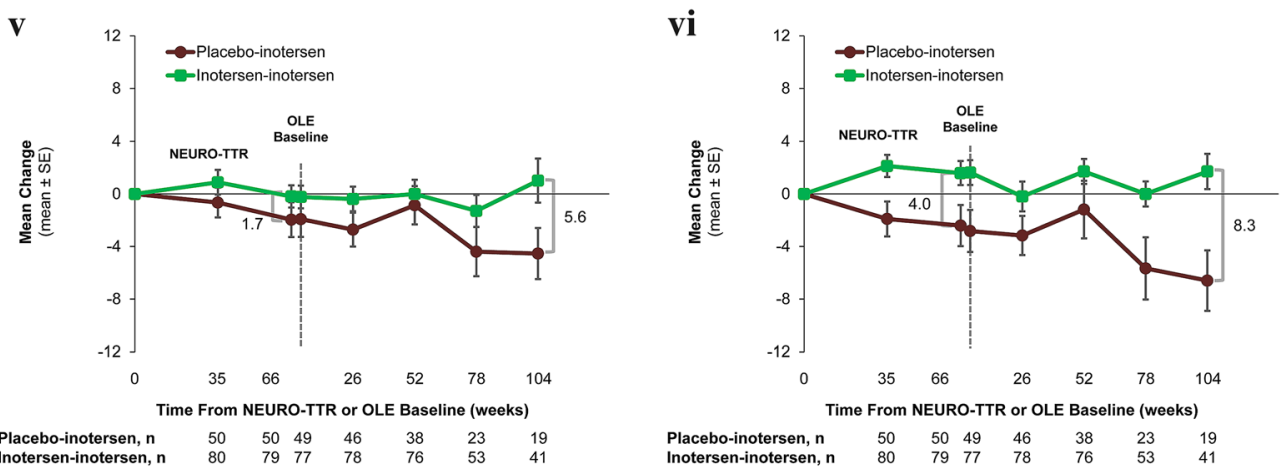
$\begin{array}{llllllll}\text { Placebo-inotersen, } \mathbf{n} & 50 & 50 & 49 & 46 & 38 & 23 & 19 \\ \text { Inotersen-inotersen, } \mathbf{n} & 80 & 79 & 77 & 78 & 76 & 53 & 41\end{array}$

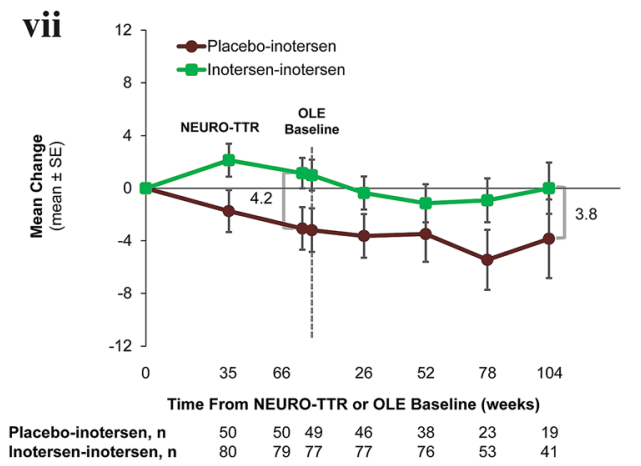

$\begin{array}{llllllll}\text { Placebo-inotersen, } \boldsymbol{n} & 50 & 50 & 49 & 46 & 38 & 23 & 19 \\ \text { Inotersen-inotersen, } \mathbf{n} & 80 & 79 & 77 & 78 & 76 & 53 & 41\end{array}$

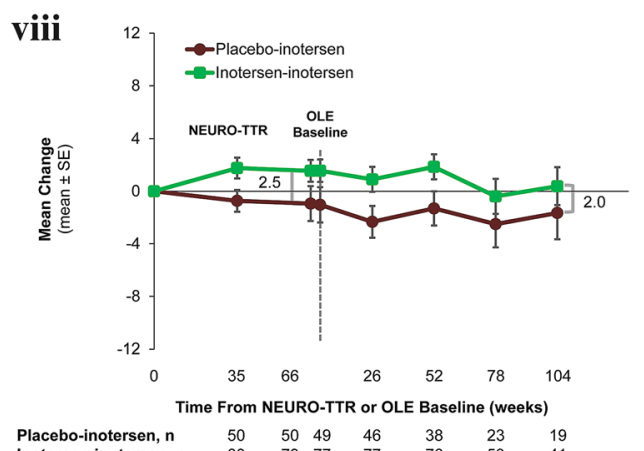

$\begin{array}{llllllll}\text { Placebo-inotersen, } \mathbf{n} & 50 & 50 & 49 & 46 & 38 & 23 & 19 \\ \text { Inotersen-inotersen, } \mathbf{n} & 80 & 79 & 77 & 77 & 76 & 53 & 41\end{array}$ 
4Fig. 2 Mean change in SF-36v2 domain scores from NEURO-TTR baseline through week 104 of the openlabel extension study. i. Physical functioning domain. ii. Role-physical domain. iii. Bodily pain domain. iv. General health domain. v. Vitality domain. vi. Social functioning domain. vii. Role-emotional domain. viii. Mental health domain. $O L E$ open-label extension, $S E$ standard error

domain). Visual inspection of trajectories for each of these items shows general stability in the proportions of patients indicating substantial impairments, with nearly all fluctuations $\leq 10$ percentage points between visits throughout the cumulative observation period.

The minimal changes of proportion of patients showing substantial impairment for the majority of selected Norfolk QOL-DN items during the combined study observation period are also evident from data reported in Table 4 . Among all observed patients at each visit, for no item was there an increase of those with substantial impairment $\geq 12.0$ percentage points from NEURO-TTR baseline to OLE week 104, while among the 41 patients who completed week 104 , for no item was there an increase $\geq 18.0$ percentage points. Furthermore, the proportion of patients indicating substantial impairment for 13 of the 19 items increased $\leq 5.3$ percentage points among all observed patients and $\leq 10.0$ percentage points among week 104 completers. Items showing the largest increases in the proportion of patients showing substantial impairment in the cumulative observation period were those that assessed difficulties getting on/off the toilet, being impaired in usual activities, and feeling unsteady while walking, all with an increase of 17.1 percentage points for week 104 completers.

\section{SF-36v2 Items}

The percentages of patients in the inoterseninotersen group indicating substantial impairment for each of the 16 selected SF-36v2 items, based on all observed item responses at each visit of the cumulative observation period, are presented in Fig. 4i (five items related to walking or climbing from the physical functioning domain), Fig. 4ii (five items related to daily activities from the physical functioning domain), Fig. 4iii (four items from the rolephysical domain), and Fig. 4iv (one item each from the bodily pain and social functioning domains). Visual inspection of trajectories for each of these items indicates general stability in the proportions of patients indicating substantial impairments, with nearly all fluctuations $\leq 10.0$ percentage points between visits across the cumulative observation period.

Table 3 Mean change in SF-36v2 domain scores from OLE baseline to week 104

\begin{tabular}{|c|c|c|c|c|}
\hline \multirow[t]{2}{*}{ Domains } & \multicolumn{2}{|c|}{ All observed scores, mean } & \multicolumn{2}{|c|}{$\begin{array}{l}\text { Week } 104 \text { completers, } \\
\text { mean }(95 \% \mathrm{CI})\end{array}$} \\
\hline & $\mathrm{I}-\mathrm{I}(n=78)$ & P-I $(n=49)$ & $\mathrm{I}-\mathrm{I}(n=41)$ & P-I $(n=19)$ \\
\hline Physical functioning & -1.6 & -0.8 & $-1.3(-4.4,1.9)$ & $-1.1(-5.8,3.7)$ \\
\hline Role-physical & -1.0 & -0.7 & $-1.1(-4.5,2.3)$ & $-1.2(-4.8,2.3)$ \\
\hline Bodily pain & 1.6 & 3.3 & $1.2(-1.4,3.8)$ & $2.4(-2.4,7.2)$ \\
\hline General health & -1.9 & -5.9 & $-0.9(-3.6,1.7)$ & $-3.6(-7.5,0.3)$ \\
\hline Vitality & 1.3 & -2.6 & $0.3(-2.4,3.0)$ & $-0.7(-5.5,4.2)$ \\
\hline Social functioning & 0.1 & -3.8 & $0.8(-1.5,3.0)$ & $-3.6(-8.5,1.3)$ \\
\hline Role-emotional & -1.0 & -0.7 & $-2.6(-6.3,1.1)$ & $-1.0(-7.3,5.4)$ \\
\hline Mental health & -1.2 & -0.6 & $-1.7(-4.2,0.8)$ & $1.5(-3.1,6.0)$ \\
\hline
\end{tabular}

$C I$ confidence interval, $I-I$ inotersen-inotersen group, $O L E$ open-label extension, $P-I$ placebo-inotersen group 

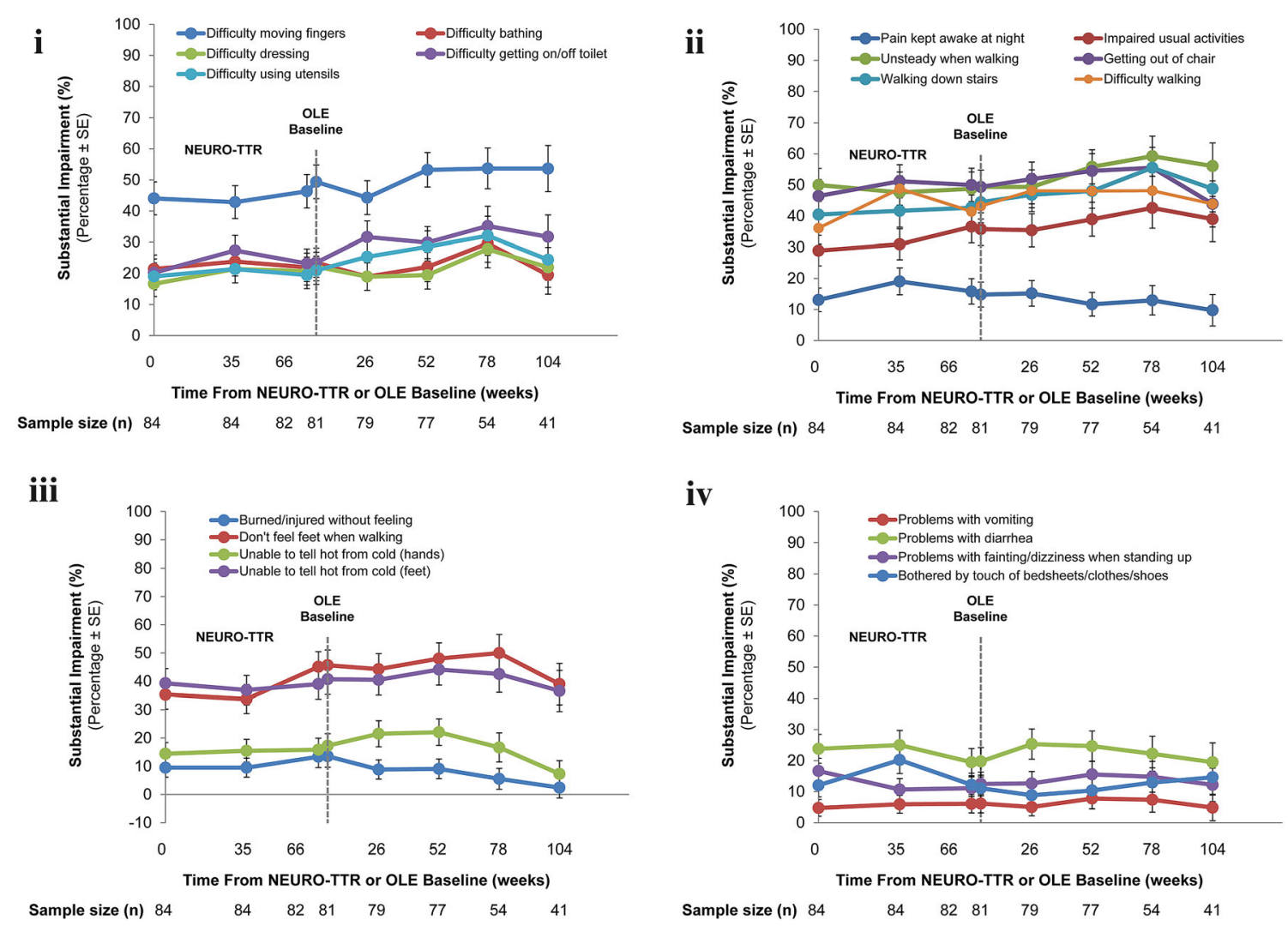

Fig. 3 Percentage of patients in the inotersen-inotersen group showing substantial impairment on Norfolk QOLDN items from NEURO-TTR baseline through week 104 of the Open-Label Extension Study. i. Items from the activities of daily living domain. ii. Items from the large

The minimal changes of proportion of patients showing substantial impairment for the majority of selected SF-36v2 items during the cumulative observation period are also evident from data reported in Table 5. Among all observed patients at each visit, for only one item was there an increase of those with substantial impairment $\geq 10.0$ percentage points between NEURO-TTR baseline and OLE week 104 assessments (an increase of 13.1 percentage points was observed for the item capturing bending/kneeling/stooping), while among the 41 subjects who completed week 104, for no item was there an increase $\geq 18.0$ percentage points. Furthermore, the proportion of patients indicating substantial impairment for 13 of the 16 items increased $\leq 5.0$ percentage points among all observed patients and for 12 items

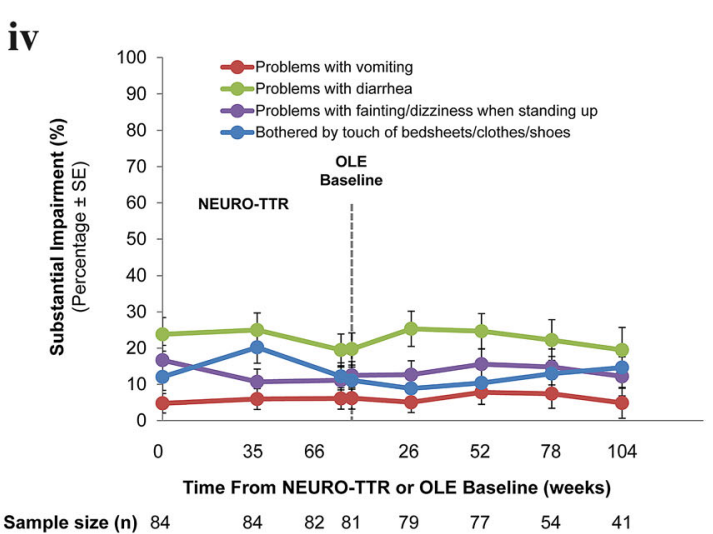

fiber neuropathy/physical functioning domain. iii. Items from the small fiber neuropathy domain. iv. Items from the autonomic neuropathy and neuropathic symptoms domains. OLE, open-label extension; SE, standard error

$\leq 10.0$ percentage points among week 104 completers. Items showing the largest increases in the proportion of patients showing substantial impairment during the cumulative observation period were those that assessed difficulties climbing one flight of stairs, climbing several flights of stairs, and in bending, walking, or stooping, with increases of 12.2, 17.1, and 14.6 percentage points, respectively, for week 104 completers.

\section{DISCUSSION}

Patients with ATTRv-PN, even in early stages of neuropathic progression, experience diminished HRQOL [5]. HRQOL, in the absence of treatment for the disease, continues to rapidly 
Table 4 Percentage of patients indicating substantial impairment in Norfolk QOL-DN items at NEURO-TTR baseline and OLE week 104

\begin{tabular}{|c|c|c|c|c|c|c|c|}
\hline \multirow[b]{2}{*}{ Domain } & \multirow[b]{2}{*}{ Item content } & \multicolumn{3}{|c|}{ All observed responses, \% } & \multicolumn{3}{|c|}{ Week 104 completers $(n=41), \%$} \\
\hline & & $\begin{array}{l}\text { NEURO-TTR } \\
\text { Baseline } \\
\quad\left(n=\mathbf{8 4}^{\mathrm{a}}\right)\end{array}$ & $\begin{array}{l}\text { Week } 104 \\
(n=41)\end{array}$ & Difference & $\begin{array}{l}\text { NEURO- } \\
\text { TTR } \\
\text { Baseline }\end{array}$ & Week 104 & Difference \\
\hline $\mathrm{ADL}$ & Difficulty moving fingers & 44.1 & 53.7 & 9.6 & 41.5 & 53.7 & 12.2 \\
\hline $\mathrm{ADL}$ & Difficulty bathing & 21.4 & 19.5 & -1.9 & 17.1 & 19.5 & 2.4 \\
\hline ADL & Difficulty dressing & 16.7 & 22.0 & 5.3 & 12.2 & 22.0 & 9.8 \\
\hline $\mathrm{ADL}$ & Difficulty getting on/off toilet & 20.2 & 31.7 & 11.5 & 14.6 & 31.7 & 17.1 \\
\hline $\mathrm{ADL}$ & Difficulty using utensils & 19.1 & 24.4 & 5.3 & 17.1 & 24.4 & 7.3 \\
\hline Autonomic & Problems with vomiting & 4.8 & 4.9 & 0.1 & 2.4 & 4.9 & 2.4 \\
\hline Autonomic & Problems with diarrhea & 23.8 & 19.5 & -4.3 & 17.1 & 19.5 & 2.4 \\
\hline Autonomic & $\begin{array}{l}\text { Problems with fainting/ } \\
\text { dizziness }\end{array}$ & 16.7 & 12.2 & -4.5 & 4.9 & 12.2 & 7.3 \\
\hline $\begin{array}{l}\text { Large fiber/ } \\
\text { PF }\end{array}$ & Pain kept awake at night & 13.1 & 9.8 & -3.3 & 17.1 & 9.8 & -7.3 \\
\hline $\begin{array}{l}\text { Large fiber/ } \\
\text { PF }\end{array}$ & Impaired usual activities & $28.9^{\mathrm{b}}$ & 39.0 & 10.1 & 22.0 & 39.0 & 17.1 \\
\hline $\begin{array}{l}\text { Large fiber/ } \\
\text { PF }\end{array}$ & Unsteady when walking & 50.0 & 56.1 & 6.1 & 39.0 & 56.1 & 17.1 \\
\hline $\begin{array}{l}\text { Large fiber/ } \\
\text { PF }\end{array}$ & Getting out of chair & 46.4 & 43.9 & -2.5 & 41.5 & 43.9 & 2.4 \\
\hline $\begin{array}{l}\text { Large fiber/ } \\
\text { PF }\end{array}$ & Walking down stairs & 40.5 & 48.8 & 8.3 & 39.0 & 48.8 & 9.8 \\
\hline $\begin{array}{l}\text { Large fiber/ } \\
\text { PF }\end{array}$ & Difficulty walking & $36.1^{\mathrm{b}}$ & 43.9 & 7.8 & $32.5^{\mathrm{d}}$ & 43.9 & 11.4 \\
\hline Small fiber & $\begin{array}{l}\text { Burned/injured without } \\
\text { feeling }\end{array}$ & 9.5 & 2.4 & -7.1 & 2.4 & 2.4 & 0.0 \\
\hline Small fiber & Don't feel feet when walking & $35.4^{\mathrm{c}}$ & 39.0 & 3.7 & 24.4 & 39.0 & 14.6 \\
\hline Small fiber & $\begin{array}{l}\text { Unable to tell hot from cold } \\
\text { (hands) }\end{array}$ & $14.5^{\mathrm{b}}$ & 7.3 & -7.1 & 9.8 & 7.3 & -2.4 \\
\hline Small fiber & $\begin{array}{l}\text { Unable to tell hot from cold } \\
\text { (feet) }\end{array}$ & 39.3 & 36.6 & -2.7 & 34.2 & 36.6 & 2.4 \\
\hline Symptoms & $\begin{array}{l}\text { Bothered by } \\
\text { bedsheets/clothes/shoes }\end{array}$ & $12.1^{\mathrm{b}}$ & 14.6 & 2.6 & 14.6 & 14.6 & 0.0 \\
\hline
\end{tabular}

$A D L$ activities of daily living, $O L E$ open-label extension, $P F$ physical functioning

a All item responses were missing for one patient

b $n=83$; an item response was missing for two patients

c $n=82$; an item response was missing for three patients

d $n=40$; an item response was missing for one patient 
deteriorate as the disease progresses [5, 8, 9]. Studies have demonstrated that recently developed, regulatory-approved gene silencing therapies indicated for patients with ATTRv-PN can preserve or even slightly improve HRQOL over periods ranging from 15 to 18 months $[12,19]$. However, to this point there is a lack of evidence as to whether these treatments are capable of arresting declines in specific aspects of HRQOL in these patients over longer durations, including those extending as far as out as 39 months (3.3 years). The data reported here provide a preliminary indication that inotersen stabilized most assessed aspects of HRQOL over this extended period of time.

A key finding from these analyses was that the gap in HRQOL between patients receiving inotersen and placebo at the end of the NEURO-
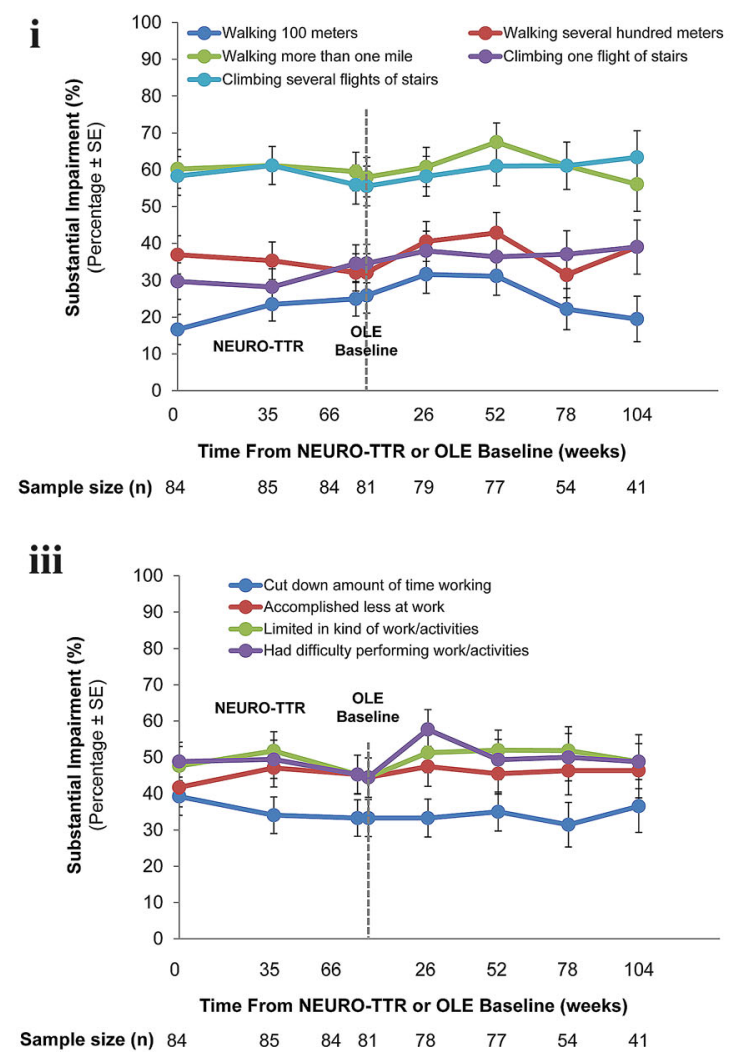

Fig. 4 Percentage of patients in the inotersen-inotersen group showing substantial impairment on SF-36v2 items from NEURO-TTR baseline through week 104 of the Open-Label Extension Study. i. Items on walking/climbing from the physical functioning domain. ii. Items on daily
TTR study remained virtually unchanged through week 104 of the OLE study for the majority of Norfolk QOL-DN and SF-36v2 domains (with the gap widening for the small fiber neuropathy domain of the Norfolk QOL$\mathrm{DN}$ and the general health, vitality, and social functioning domains of the SF-36v2), with nearly parallel horizontal lines for scores on graphs depicting mean change over time. All current gene-silencing drugs that have been approved by regulatory bodies for treatment of patients with ATTRv-PN, including inotersen, effectively suppress production of plasma TTR, thus preventing new amyloid from accumulating in the tissues in organs. However, these treatments do not remove amyloid that has already been stored in these tissues and organs. So, while inotersen can stop further damage
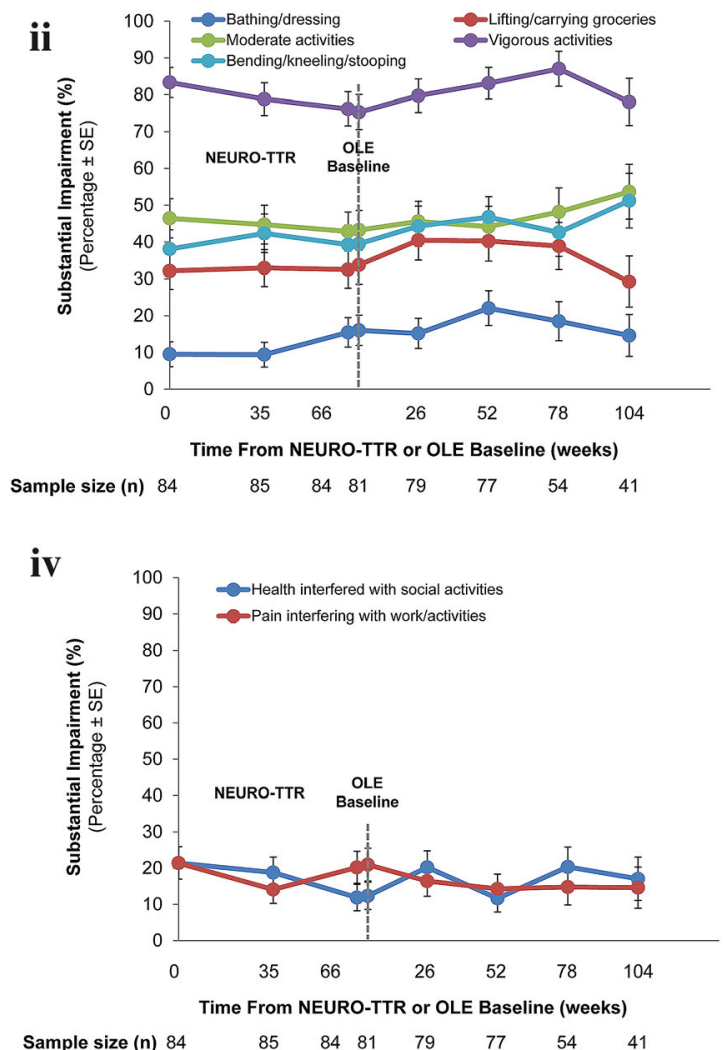

activities from the physical functioning domain. iii. Items from the role-physical domain. iv. Items from the bodily pain and social functioning domains. OLE open-label extension, $S E$ standard error 
Table 5 Percentage of patients indicating substantial impairment in SF-36v2 items at NEURO-TTR baseline and OLE week 104

\begin{tabular}{|c|c|c|c|c|c|c|c|}
\hline \multirow[t]{2}{*}{ Domain } & \multirow[t]{2}{*}{ Item content } & \multicolumn{3}{|c|}{ All observed responses, $\%$} & \multicolumn{3}{|c|}{ Week 104 completers $(n=41), \%$} \\
\hline & & $\begin{array}{l}\text { NEURO- } \\
\text { TTR } \\
\text { Baseline } \\
\quad\left(n=\mathbf{8 4}^{\mathrm{a}}\right)\end{array}$ & $\begin{array}{l}\text { Week } 104 \\
(n=41)\end{array}$ & Difference & $\begin{array}{l}\text { NEURO- } \\
\text { TTR } \\
\text { Baseline }\end{array}$ & Week 104 & Difference \\
\hline $\begin{array}{l}\text { Physical } \\
\text { functioning }\end{array}$ & Walking $100 \mathrm{~m}$ & 16.7 & 19.5 & 2.8 & 12.2 & 19.5 & 7.3 \\
\hline $\begin{array}{l}\text { Physical } \\
\text { functioning }\end{array}$ & $\begin{array}{l}\text { Walking several hundred } \\
\text { meters }\end{array}$ & 36.9 & 39.0 & 2.1 & 26.8 & 39.0 & 12.2 \\
\hline $\begin{array}{l}\text { Physical } \\
\text { functioning }\end{array}$ & Walking more than 1 mile & 60.2 & 56.1 & -4.1 & 51.2 & 56.1 & 4.9 \\
\hline $\begin{array}{l}\text { Physical } \\
\text { functioning }\end{array}$ & Climbing one flight of stairs & 29.8 & 39.0 & 9.3 & 26.8 & 39.0 & 12.2 \\
\hline $\begin{array}{l}\text { Physical } \\
\text { functioning }\end{array}$ & $\begin{array}{l}\text { Climbing several flights of } \\
\text { stairs }\end{array}$ & 58.3 & 63.4 & 5.1 & 46.3 & 63.4 & 17.1 \\
\hline $\begin{array}{l}\text { Physical } \\
\text { functioning }\end{array}$ & Bathing/dressing & 9.5 & 14.6 & 5.1 & 9.8 & 14.6 & 4.9 \\
\hline $\begin{array}{l}\text { Physical } \\
\text { functioning }\end{array}$ & Lifting/carrying groceries & 32.1 & 29.3 & -2.9 & 26.8 & 29.3 & 2.4 \\
\hline $\begin{array}{l}\text { Physical } \\
\text { functioning }\end{array}$ & Moderate activities & 46.4 & 53.7 & 7.2 & 46.3 & 53.7 & 7.3 \\
\hline $\begin{array}{l}\text { Physical } \\
\text { functioning }\end{array}$ & Vigorous activities & 83.3 & 78.1 & -5.3 & 80.5 & 78.1 & -2.4 \\
\hline $\begin{array}{l}\text { Physical } \\
\text { functioning }\end{array}$ & Bending/kneeling/stooping & 38.1 & 51.2 & 13.1 & 36.6 & 51.2 & 14.6 \\
\hline Role-physical & Cutting down time working & 39.3 & 36.6 & -2.7 & 34.2 & 36.6 & 2.4 \\
\hline Role-physical & Accomplished less at work & 41.7 & 46.3 & 4.7 & 43.9 & 46.3 & 2.4 \\
\hline Role-physical & $\begin{array}{l}\text { Limited in kind of work/ } \\
\text { activities }\end{array}$ & 47.6 & 48.8 & 1.2 & 46.3 & 48.8 & 2.4 \\
\hline Role-physical & $\begin{array}{l}\text { Difficulty performing work/ } \\
\text { activities }\end{array}$ & 48.8 & 48.8 & 0.0 & 43.9 & 48.8 & 4.9 \\
\hline Bodily pain & $\begin{array}{l}\text { Pain interfered with work/ } \\
\text { activities }\end{array}$ & 21.4 & 14.6 & -6.8 & 22.0 & 14.6 & -7.3 \\
\hline $\begin{array}{l}\text { Social } \\
\text { functioning }\end{array}$ & $\begin{array}{l}\text { Health interfered with social } \\
\text { activities }\end{array}$ & 21.4 & 17.1 & -4.4 & 17.1 & 17.1 & 0.0 \\
\hline
\end{tabular}

$O L E$ open-label extension

a All item responses were missing for one patient 
from occurring because of new amyloid, it cannot reverse the damage that occurred prior to treatment, including the subsequent impacts of this damage on patients' quality of life. These results stress the importance of early diagnosis and treatment, though they still support later treatment even at more advanced states.

As mentioned above, there were some domains for which the gaps between mean changes in inotersen-inotersen and placeboinotersen groups increased during the course of the OLE study, with all of these increases driven by the start or continuation of deterioration in the latter group during the OLE observational period. While neither group showed progression during the NEURO-TTR study on the Norfolk QOL-DN small fiber neuropathy domain, patients in the placebo-inotersen group began showing progression during the OLE study, with the increase initially observed at week 26 and then remaining throughout the OLE observation period. However, the magnitude of progression is relatively small: at both week 26 and week 104 visits of the OLE study, the increase from NEURO-TTR baseline was only 1.4 points, and the difference with the inoterseninotersen group never exceeded 1 point during the OLE observation period, with overlapping standard errors between the two groups at all visits. Thus, it appears unlikely that this magnitude represents a clinically meaningful change in small fiber neuropathy for patients in this group.

The same cannot be said for the three SF36v2 domains-general health, vitality, and social functioning-for which the placeboinotersen group showed substantial worsening during the OLE observation period. Patterns of deterioration for this group were similar for all three domains, with little change observed through week 52 , followed by a rapid decline at the week 78 visit which carried over to the week 104 visit. For general health and vitality domains, the between-group difference in mean change more than tripled from the end of the NEURO-TTR study to week 104 of the OLE study, while for social functioning the gap doubled in size. The explanation for these considerable declines after week 52 of the OLE study is not immediately apparent, particularly for the general health and vitality domains, which showed relatively small declines even when patients received only placebo during the NEURO-TTR study. OLE studies of patients with ATTRv who received other treatments, such as patisiran (NCT02510261) or tafamidis (NCT02791230), did not include the SF-36v2 as an endpoint, so no data are available for comparison. It is hoped that future research, in particular evaluation of SF-36v2 scores within a more recent cut of the data from the OLE study, for which there will be several more patients completing assessment at week 78 and week 104 visits, will provide more precise data regarding long-term impact of inotersen on these outcomes.

There are several limitations of this study that warrant using caution when interpreting these findings. First, these analyses were conducted using an interim, rather than completed, dataset from the OLE study, for which data were available for only 60 (44.4\%) enrolled patients at the week 104 visit. At the time that these data were cut, $33(24.4 \%)$ patients receiving ongoing treatment in the OLE study had not yet completed the week 104 visit. Furthermore, $42(31.1 \%)$ patients who enrolled in the OLE study-27 patients (31.7\%) in the inotersen-inotersen group and 15 patients $(30.0 \%)$ in the placebo-inotersen group-discontinued treatment prior to the week 104 visit. While patient discontinuations and other results from analysis of safety in the OLE study have been reported previously [13], it is worth noting here that adverse events (AEs) or serious $\mathrm{AE}$ (SAEs) were recorded as the reason for discontinuation for more than half of the discontinuations in the inotersen-inotersen group (15, or $55.6 \%$ ) compared to a much smaller proportion of discontinuations in the placebo-inotersen group (3, or $20.0 \%)$. At the same time, only a minority of the AEs/SAEs that led to a patient discontinuation were treatment-related (4 for the inotersen-inotersen group and 1 for the placebo-inotersen group). Furthermore, all fatal treatment-emergent AEs in the OLE were determined to be unrelated to study treatment. Still, while it was concluded that data from the OLE provide support for the long-term safety of inotersen [13], the impact of these 
discontinuations on sample sizes at later visits in the OLE observation period is sizable. The analysis of patients' HRQOL at these later visits should therefore be considered preliminary and may be impacted by survivor bias, such that HRQOL observed for patients at this visit may overestimate that seen in the broader population of ATTRV-PN patients receiving inotersen. Furthermore, this resulted in very small samples that limited the ability to test longitudinal models that could have provided an opportunity to adjust for relevant patient or disease characteristics or to test the statistical significance for differences in mean scores either between or within patient groups. As such, the findings presented here are qualitative in nature. Another limitation due to small sample size was that it did not allow for examining changes in quality of life within and across subgroups of patients defined by key patients or clinical characteristics, such as early or late onset of disease symptoms. To address many of these limitations, future analyses will be conducted to re-examine the updated dataset once all continuing patients have completed the week 104 visit of the OLE study.

Other limitations were direct consequences of the OLE study design, for which, by definition, all patients were treated with inotersen in an open-label manner. The lack of random allocation of patients to treatment and the absence of a control group prevent the ability to make causal inferences from these findings. Furthermore, all patients in the OLE study were aware that they were receiving inotersen treatment throughout the study. This knowledge may have impacted patients' perceptions of their own health status, which could then bias their responses to self-reported HRQOL assessments such as those used here.

\section{CONCLUSIONS}

In conclusion, the findings presented here, while preliminary, suggest that continuous treatment with inotersen may preserve HRQOL of patients with ATTRv-PN for periods of up to 3 years. Given that previous analyses observed no additional safety concerns or increased toxicity in patients exposed to inotersen for up to 5 years [13], inotersen may be a promising long-term treatment option for these patients. Importantly, these findings also indicate that while deterioration of HRQOL for these patients can be arrested by inotersen, it was not reversed, which highlights the importance of early diagnosis and treatment of this disease.

\section{ACKNOWLEDGEMENTS}

We thank the patients who participated in this trial and their families.

Funding. This research, as well as the journal's Rapid Service Fee and Open Access Fee, was funded by Akcea Therapeutics, a subsidiary of Ionis Pharmaceuticals Inc.

Editorial Assistance. We thank Bonita S. Basnyat of QualityMetric for copy editing and preparation of this manuscript for submission, for which QualityMetric was funded by Akcea Therapeutics, a subsidiary of Ionis Pharmaceuticals Inc.

Authorship. All named authors meet the International Committee of Medical Journal Editors (ICMJE) criteria for authorship for this article, take responsibility for the integrity of the work as a whole, and have given their approval for this version to be published.

Author Contributions. Aaron Yarlas contributed to the study design, conducted statistical analysis, drafted the first and final drafts of the manuscript, and approved the final manuscript. Andrew Lovley contributed to the study design, conducted statistical analysis, commented on previous versions of the manuscript, and read and approved the final manuscript. Kristen McCausland contributed to the study design, commented on previous versions of the manuscript, and read and approved the final manuscript. Duncan Brown contributed to the study design, commented on previous versions of the manuscript, and read and approved the final manuscript. Montserrat Vera-Llonch 
contributed to the study design, commented on previous versions of the manuscript, and read and approved the final manuscript. Isabel Conceição contributed to the study design, commented on previous versions of the manuscript, and read and approved the final manuscript. Chafic Karam contributed to the study design, commented on previous versions of the manuscript, and read and approved the final manuscript. Sami Khella contributed to the study design, commented on previous versions of the manuscript, and read and approved the final manuscript. Laura Obici contributed to the study design, commented on previous versions of the manuscript, and read and approved the final manuscript. Márcia Waddington-Cruz contributed to the study design, commented on previous versions of the manuscript, and read and approved the final manuscript.

List of Principal Investigators. Principal Investigators for the NEURO-TTR open-label extension study were as follows: David Adams, MD, PhD, Paris, France. Fabio A Barroso, MD, Buenos Aires, Argentina. Merrill D Benson, MD, Indianapolis, IN, USA. John L Berk, MD, Boston, MA, USA. Thomas H Brannagan III, MD, New York, NY, USA. Josep Maria Campistol Plana, MD, Barcelona, Spain. Jose Gamez Carbonell, MD, PhD, Barcelona, Spain. Teresa Coelho, MD, Porto, Portugal. Isabel Conceição, MD, Lisbon, Portugal. Brian M Drachman, MD, Philadelphia, PA, USA. Edward Gane, MD, Auckland, New Zealand. Morie A Gertz, MD, Rochester, MN. Peter D Gorevic, MD, New York, NY. Stephen B Heitner, MD, Portland, OR. Arnt Kristen, Heidelberg, Germany. Giampaolo Merlini, MD, Pavia, Italy. Laura Obici, MD, Pavia, Italy. Violaine Plante-Bordeneuve, MD, Créteil, France. Michael Polydefkis, MD, MHS, Baltimore, MD. Fabrizio Salvi, MD, Bologna, Italy. Morton Scheinberg, MD, São Paulo, Brazil. Hartmut H Schmidt, MD, Muenster, Germany. Acary Souza Bulle Oliveira, MD, São Paulo, Brazil. Giuseppe Vita, MD, Messina, Italy. Annabel K Wang, MD, Irvine, CA. Márcia Waddington-Cruz, MD, PhD, Rio de Janeiro, Brazil. Carol Whelan, MD. London, UK.
Prior Presentation. Portions of this research were presented at the following conferences: American Academy of Neurology (AAN) Annual Meeting, April 17-22, 2020, Virtual; XVII International Symposium on Amyloidosis (ISA), September 1-18, 2020, Virtual; and American Association of Neuromuscular \& Electrodiagnostic Medicine (AANEM) Annual Meeting, October 7-10, 2020, Virtual.

Disclosures. Aaron Yarlas, Andrew Lovley, and Kristen McCausland are employees of QualityMetric, which received payment from Ionis Pharmaceuticals to conduct these analyses and develop this manuscript. Duncan Brown and Montserrat Vera-Llonch are employees of and own stock in Ionis Pharmaceuticals. Isabel Conceição acknowledges financial support as a primary investigator for FoldRx Pharmaceuticals/Pfizer, Alnylam Pharmaceuticals, and IONIS Pharmaceuticals, and serves on the Transthyretin Amyloidosis Outcome Survey (THAOS) scientific advisory board. Chafic Karam has received consulting honoraria from Argenx, CSLBehring, Alnylam, Akcea Therapeutics, Biogen, and Sanofi. Sami Khella has received consulting honoraria from Ionis Pharmaceuticals, Akcea Therapeutics, Alnylam, Pfizer, and Eidos. Laura Obici has received speaker honoraria from Akcea, Pfizer, and Alnylam. Márcia Waddington-Cruz has received honoraria from NHI, Prothena, FoldRx, Akcea Therapeutics, Pfizer, Alnylam Pharmaceuticals, PTC, and Genzyme for travel expenses related to presentations at medical meetings, and for acting as a consultant and as a principal investigator in clinical trials.

Compliance with Ethics Guidelines. The trial protocol was approved by institutional review boards or local ethics committees. The trial was conducted in accordance with Good Clinical Practice guidelines of the International Conference on Harmonisation and the principles of the Declaration of Helsinki of 1964 and its later amendments. Written informed consent was obtained from all participants included in the study. 
Data Availability. Data can be made available upon reasonable request.

Open Access. This article is licensed under a Creative Commons Attribution-NonCommercial 4.0 International License, which permits any non-commercial use, sharing, adaptation, distribution and reproduction in any medium or format, as long as you give appropriate credit to the original author(s) and the source, provide a link to the Creative Commons licence, and indicate if changes were made. The images or other third party material in this article are included in the article's Creative Commons licence, unless indicated otherwise in a credit line to the material. If material is not included in the article's Creative Commons licence and your intended use is not permitted by statutory regulation or exceeds the permitted use, you will need to obtain permission directly from the copyright holder. To view a copy of this licence, visit http://creativecommons.org/licenses/by$\mathrm{nc} / 4.0 /$.

\section{REFERENCES}

1. Hawkins PN, Ando Y, Dispenzeri A, GonzalezDuarte A, Adams D, Suhr OB. Evolving landscape in the management of transthyretin amyloidosis. Ann Med. 2015;47:625-38. https://doi.org/10.3109/ 07853890.2015 .1068949 .

2. Ando Y, Coelho T, Berk JL, Cruz MW, Ericzon B-G, Ikeda $S$, et al. Guideline of transthyretin-related hereditary amyloidosis for clinicians. Orphanet J Rare Dis. 2013;8:31. https://doi.org/10.1186/17501172-8-31.

3. Rowczenio DM, Noor I, Gillmore JD, Lachmann HJ, Whelan C, Hawkins PN, et al. Online registry for mutations in hereditary amyloidosis including nomenclature recommendations. Hum Mutat. 2014;35:E2403-12. https://doi.org/10.1002/humu. 22619.

4. Sekijima Y. Transthyretin (ATTR) amyloidosis: clinical spectrum, molecular pathogenesis and disease-modifying treatments. J Neurol Neurosurg Psychiatry. 2015;86:1036-43. https://doi.org/10. 1136/jnnp-2014-308724.

5. Yarlas A, Gertz MA, Dasgupta NR, Obici L, Pollock $\mathrm{M}$, Ackermann EJ, et al. Burden of hereditary transthyretin amyloidosis on quality of life. Muscle Nerve. 2019;60:169-75. https://doi.org/10.1002/ mus. 26515 .

6. Stewart M, Shaffer S, Murphy B, Loftus J, Alvir J, Cicchetti M, Lenderking WR. Characterizing the high disease burden of transthyretin amyloidosis for patients and caregivers. Neurol Ther. 2018. https://doi.org/10.1007/s40120-018-0106-z.

7. Lovley A, Raymond K, Guthrie SD, Pollock M, Sanchorawala V, White MK. Patient-reported burden of hereditary transthyretin amyloidosis on functioning and well-being. J Patient-Rep Outcomes. 2021;5:3. https://doi.org/10.1186/s41687020-00273-y.

8. Coelho T, Vinik A, Vinik EJ, Tripp T, Packman J, Grogan DR. Clinical measures in transthyretin familial amyloid polyneuropathy. Muscle Nerve. 2017;55:323-32. https://doi.org/10.1002/mus. 25257.

9. Inês M, Coelho T, Conceição I, Ferreira L, de Carvalho $\mathrm{M}$, Costa J. Health-related quality of life in hereditary transthyretin amyloidosis polyneuropathy: a prospective, observational study. Orphanet J Rare Dis. 2020;15:67. https://doi.org/10.1186/ s13023-020-1340-x.

10. Benson MD, Waddington-Cruz M, Berk JL, Polydefkis M, Dyck PJ, Wang AK, et al. Inotersen treatment for patients with hereditary transthyretin amyloidosis. N Engl J Med. 2018;379:22-31. https:// doi.org/10.1056/NEJMoa1716793.

11. Dyck P, James B, Coelho T, Waddington Cruz M, Brannagan TH, Khella S, Karam C, et al. Neuropathy symptom and change: Inotersen treatment of hereditary transthyretin amyloidosis. Muscle Nerve. 2020;62:509-15. https://doi.org/10.1002/ mus. 27023 .

12. Coelho T, Yarlas A, Waddington-Cruz M, White MK, Sikora Kessler A, Lovley A, et al. Inotersen preserves or improves quality of life in hereditary transthyretin amyloidosis. J Neurol. 2020;267: 1070-9. https://doi.org/10.1007/s00415-01909671-9.

13. Brannagan $\mathrm{TH}$, Wang $\mathrm{AK}$, Coelho $\mathrm{T}$, Waddington Cruz M, Polydefkis MJ, Dyck PJ, et al. Early data on long-term efficacy and safety of inotersen in patients with hereditary transthyretin amyloidosis: a 2-year update from the open-label extension of the NEURO-TTR trial. Eur J Neurol. 2020;27: 1374-81. https://doi.org/10.1111/ene.14285.

14. Coutinho P, Martins da Silva A, Lopes Lima J, Resende Barbosa A. Forty years of experience with type 1 amyloid neuropathy. Review of 483 cases. In: Glenner G, Costa P, Freitas A de, editors. Amyloid 
and Amyloidosis. Amsterdam: Execerpta Medica; 1980. p. 88-98.

15. Vinik EJ, Hayes RP, Oglesby A, Bastyr E, Barlow P, Ford-Molvik SL, Vinik AI. The development and validation of the Norfolk QOL-DN, a new measure of patients' perception of the effects of diabetes and diabetic neuropathy. Diabetes Technol Ther. 2005;7:497-508. https://doi.org/10.1089/dia.2005. 7.497.

16. Vinik EJ, Vinik AI, Paulson JF, Merkies ISJ, Packman J, Grogan DR, Coelho T. Norfolk QOL-DN: validation of a patient reported outcome measure in transthyretin familial amyloid polyneuropathy. J Peripher Nerv Syst. 2014;19:104-14. https://doi. org/10.1111/jns5.12059.
17. Maruish ME. User's manual for the SF-36v2 health survey. 3rd ed. Lincoln, RI: QualityMetric Incorporated; 2011.

18. Berk JL, Suhr OB, Obici L, Sekijima Y, Zeldenrust SR, Yamashita $\mathrm{T}$, et al. Repurposing diflunisal for familial amyloid polyneuropathy: a randomized clinical trial. JAMA. 2013;310:2658-67. https://doi. org/10.1001/jama.2013.283815.

19. Obici L, Berk JL, González-Duarte A, Coelho T, Gillmore J, Schmidt HH-J, et al. Quality of life outcomes in APOLLO, the phase 3 trial of the RNAi therapeutic patisiran in patients with hereditary transthyretin-mediated amyloidosis. Amyloid. 2020;27:153-62. 13506129.2020 .1730790 . 